\title{
Fundamentos e agenda da inclusão digital no Brasil: pesquisas em Educação
}

\author{
The background and agenda of digital inclusion in Brazil: \\ research in Education
}

\author{
Fundamentos y agenda de inclusión digital en Brasil: \\ investigación en Educación
}

\author{
BARBARA COELHO NEVES* \\ EDVALDO SOUZA COUTO** \\ MARIA COUTO CUNHA***
}

\begin{abstract}
RESUMO - O artigo apresenta resultados parciais de uma investigação acerca dos fundamentos dos estudos e pesquisas desenvolvidos no Brasil sobre inclusão digital na área de Educação e apresenta os fundamentos da proposição de uma agenda dessa produção acadêmica. Traz como objetivos discutir os fundamentos teóricoconceituais e metodológicos, determinar o termo agenda da inclusão digital empregado neste estudo e abordar alguns dos indicadores (clusters) levantados na pesquisa macro da qual o artigo é uma das partes complementares. O método utilizado é o infométrico, aplicado no Banco de Teses e Dissertações da Capes. A análise dos dados é realizada a partir do teste sociométrico. Como resultado, o artigo indica aspectos teóricos, metodológicos e conceituais a serem analisados no desenvolvimento da pesquisa.
\end{abstract}

Palavras-chave - Inclusão digital. Educação. Pesquisas. Estudo infométrico. Produção científica.

ABSTRACT - The article presents the results of an investigation into the foundations of the research carried on digital inclusion in Brazil in the area of Education. Presents the fundamentals of the proposition of an academic research agenda focused on digital inclusion. Has as objectives to discuss the theoretical, conceptual and methodological foundations, determine the digital inclusion agenda, term employed in this study, and present some of the indicators (clusters) uncovered by the research of macro, where the article it is complementary part. The method used is the infometric developed at the Capes dissertations theses data-banks. Data analysis is performed from the sociometric test. As a result the article indicates aspects theoretical, methodological and conceptual to be analyzed in the research development.

Keywords - Digital inclusion. Education. Searches. Infometric study. Scientific pruduction.

RESUMEN - El artículo presenta los resultados de una investigación sobre los fundamentos de la realizada sobre la inclusión digital en Brasil en el área de Educación. Presenta los fundamentos de una agenda que la investigación académica se centró en la inclusión digital. Tiene como objetivos para discutir los fundamentos teoricos, conceptuales y metodologicos, determinar la agenda del termo (inclusión digital) empleado en este estudio y presentar algunos de los indicadores (clusters) descubiertos en la investigación macro donde este articulo es un producto de los componentes que emparamentan. El método utilizado es el infometric, desarrollado en el Banco de Tesis y Disertaciones de la Capes. El análisis de datos se realiza a partir de la prueba sociométric. Como resultado, el article indica conceptos teóricos, metodologicos que deben ser analizadas y en la Investigación.

Palabras clave - Inclución digital. Educación. Investigación. Estudio infometric. Produción cientifica.

\footnotetext{
*Doutora em Educação pela Universidade Federal da Bahia (Salvador, BA, Brasil) e professora na Universidade Federal de Sergipe (São Cristóvão, SE, Brasil).E-mail: <barbaracoelho@ufs.br>.

**Doutor em Educação pela Universidade Estadual de Campinas (Campinas, SP, Brasil) e professor na Universidade Federal da Bahia (Salvador, BA, Brasil) E-mail: <edvaldosouzacouto@gmail.com>.

*** Doutora em Educação pela Universidade Federal da Bahia (Salvador, BA, Brasil) e professora na Universidade Federal da Bahia (Salvador, BA, Brasil). E-mail: <mariacoutocunha@gmail.com>.
} 


\section{INTRODUÇÃO}

Inicialmente, a expressão fundamentos de estudos e pesquisas é empregada para indicar que se trata de uma pesquisa analítica, que busca aprofundar os aspectos inscritos nas teses e dissertações sobre inclusão digital, defendidas ao longo dos anos 2000, na área de Educação. Os fundamentos que os autores utilizam para construir sua resposta de pesquisa são a preocupação deste artigo.

O termo agenda, também destacado neste estudo, é apresentado com dois significados. Em primeiro lugar, por se tratar de uma pesquisa que aborda a inclusão digital, a palavra agenda geralmente corresponde ao conjunto das políticas públicas de um determinado período e voltadas a uma questão social específica. Entende-se inclusão digital como categoria política e, desse modo, o estudo deve perpassar pelas políticas públicas de inclusão digital que influenciam a educação. Nesse caso, as políticas públicas são "pano de fundo" em algumas discussões das possíveis respostas que aqui se apresentam. Em segundo lugar, a palavra agenda diz respeito ao resultado que a tese proposta, resultante da pesquisa em andamento, pretende tratar para se somar aos estudos que discutem a temática da inclusão digital.

Nesse contexto, a investigação trata de um acompanhamento e de uma análise da agenda que constitui as pesquisas produzidas sobre inclusão digital na Educação brasileira. Parte-se do pressuposto de que o crescente interesse dos pesquisadores da Educação por esse tema está diretamente relacionado às mudanças recentes da sociedade brasileira. Respectivamente, as novas questões da agenda política brasileira constituem também um problema para o desenvolvimento da agenda de pesquisa em inclusão digital.

As políticas públicas estão associadas às políticas sociais que, por sua vez, recentemente são construídas a partir da promulgação da Constituição Federal (CF) de 1988. Desse modo, compreende-se que a agenda de pesquisa é determinada, também, pelas políticas públicas. Os sujeitos que estudam a inclusão digital na Educação estão preocupados com as formas de acesso, treinamento e formação que envolvem os sujeitos pesquisados com as políticas públicas, os programas e projetos de inclusão digital, como também como se dão esses aspectos no contexto escolar. Feitos esses esclarecimentos, inicia-se a discussão do objeto desta investigação.

O que se vislumbra, atualmente, no âmbito da Educação brasileira, com relação à inclusão digital, é o resultado, principalmente, das transformações que envolvem tecnologias da informação e da comunicação (TICs) nessa área, que procedem, de maneira mais intensa, desde o início dos anos 2000. Desse modo, observar e analisar os objetos de discussão, como são fundamentados e sob quais lentes paradigmáticas são abordados na literatura é uma maneira de reconstruir o caminho desse tema na área da Educação e da Comunicação. Assim como também é uma forma de olhar para o futuro, aproximando-se de elementos e aspectos do objeto que permitem traçar novas agendas para lidar com as transformações das relações humanas em curso.

\section{EDUCAÇÃO E INCLUSÃO DIGITAL: O CONTEXTO E A PROBLEMÁTICA}

Compreende-se a inclusão digital como uma categoria de análise, sendo esta "para si" um objeto político. Trata-se de um discurso político materializado em programas, linhas e estratégias de ação, projetos e centros sociodigitais. Enquanto categoria, a inclusão digital vem sendo observada na Educação como política pública. O principal contexto dos sujeitos, sobre os quais se desenvolvem as análises nessa área, tem sido a escola.

No Brasil, a inclusão digital, na maioria das vezes, é um discurso político apropriado pelos governos para o desenvolvimento de programas e linhas de ação. Isso quer dizer que parcelas da sociedade, a partir do discurso dos governantes, têm uma "noção" dessa inclusão. Vislumbram uma compreensão generalista (do ponto de vista conceitual) e restrita (do ponto de vista da ação) com relação ao acesso às tecnologias, sobretudo no que diz respeito aos computadores e à internet. Esse aspecto é o que Dijk (2006) chama a atenção, ressaltando que o discurso político é muito mais poderoso e predominante quando se trata do par dialético exclusão e inclusão digital. O que se observa é que não há um conceito, mas sim uma série de elementos que apontam perspectivas que se baseiam em propostas voltadas para o acesso, treinamento ou formação. Partindo disso, este estudo compreende a inclusão digital como um contexto, movimento social, da atual demanda de formação dos indivíduos ante as tecnologias na sociedade contemporânea.

Com base nesses aspectos, percebe-se a ausência de trabalhos que mapeiem, observem e discutam, simultaneamente, o comportamento e as tendências teóricas, metodológicas e conceituais que fundamentam o tema inclusão digital na produção da pós-graduação em Educação. Nas cercanias desse problema, um levantamento prévio na Base de Teses e Dissertações da Capes, mantido pelo Ministério da Educação, mostrou que existe uma preocupação crescente com esse tema nas diversas áreas do conhecimento.

\subsection{Materiais e método}

A pesquisa reúne a perspectiva de compreender a consistência do tema inclusão digital, considerando aspectos da fundamentação e pressupostos teóricos, em 
teses e dissertações defendidas nos anos 2000, na área de educação. A maior inquietação diz respeito às abordagens que sustentam as discussões sobre tecnologia no âmbito da Educação e o conceito de inclusão que se encontra por trás do termo inclusão digital. Para tanto, utiliza-se na pesquisa a infometria ${ }^{1}$, observação indireta e análise por meio da aplicação do teste sociométrico.

O estudo infométrico considera a informação registrada nos variados formatos, a exemplo do digital, do sonoro, do escrito e, em qualquer suporte material, como papel, disco, filme, banda magnética, nuvem informática, dentre outros. Talvez o maior expoente de investigação nesse sentido sejam os estudos elaborados pela American Library Asociation (ALA), e cabe destacar os escritos que tratam e definem a infometria e suas leis de aplicação, a exemplo de Vanti (2002, 2005), Guedes e Borschiver (2005). Esta possibilita levantar os estudos sobre inclusão digital na área de Educação com rigor metodológico próprio, permitindo, ao mesmo tempo, a flexibilidade de movimentar e propor análises variadas dos resultados. Isso devido às suas saídas de dados em formato de rede.

Já o estudo sociométrico consiste na elaboração de perguntas, sendo realizada a tabulação das respostas e a elaboração do sociograma. Essa atividade vem sendo feita para a representação gráfica ou pictórica da tabulação sociométrica dos indicadores que cercam os estudos levantados. A técnica sociométrica e o sociograma (que é a sua representação gráfica) admitem verificar como se apresentam as relações sociais entre os pesquisadores e distinguir os líderes aceitos nos grupos (clusters).

Sua problemática está na junção da infometria (método para abordagem quantitativa) e da sociometria (análise qualitativa), considerando o tema inclusão digital na Educação. Assim, consequentemente, as temáticas principais que envolvem o tema em foco nas teses e dissertações, que se constituem objetos desta análise, também perpassam as preocupações das discussões aqui levantadas. Deste modo, para compreender as incidências do tema inclusão digital na pós-graduação na área de Educação, foi necessário realizar um mapeamento dessa produção. Para traçar tal mapa, fez-se uso da aplicação de métodos estatísticos específicos, sendo a infometria associada à sociometria, sob nossa perspectiva, como os mais adequados.

Com base em autores como Warschauer (2006a, 2006b), observa-se que a educação e o aprendizado se constituem um viés preponderante na construção de uma sociedade da informação, de acordo com os moldes do atual contexto socioeconômico, baseandose nos novos meios de entretenimento, relacionamento, empregabilidade, consumo e formação de identidades.

\subsection{Enfoque fotográfico: os números da pesquisa na área de Educação}

Vale salientar que, quando se iniciou a pesquisa, o intuito era levantar em campo documentos (teses e dissertações) do período que compreendia desde o ano 2000 até os dias atuais. Ao se iniciarem os procedimentos no Banco de Teses e Dissertações da Capes, no início do ano de 2013, percebeu-se que estavam disponíveis documentos até 2010. Desse modo, este artigo traz resultados balizados em um marco temporal que compreende a primeira etapa da pesquisa, qual seja os anos compreendidos entre 2000 e 2010.

O Banco de Teses e Dissertações da Capes apresenta o volume de trabalhos sobre o tema inclusão digital, demonstrado na Figura 1, apontando o número total de teses defendidas sobre essa temática nas diversas áreas do conhecimento.

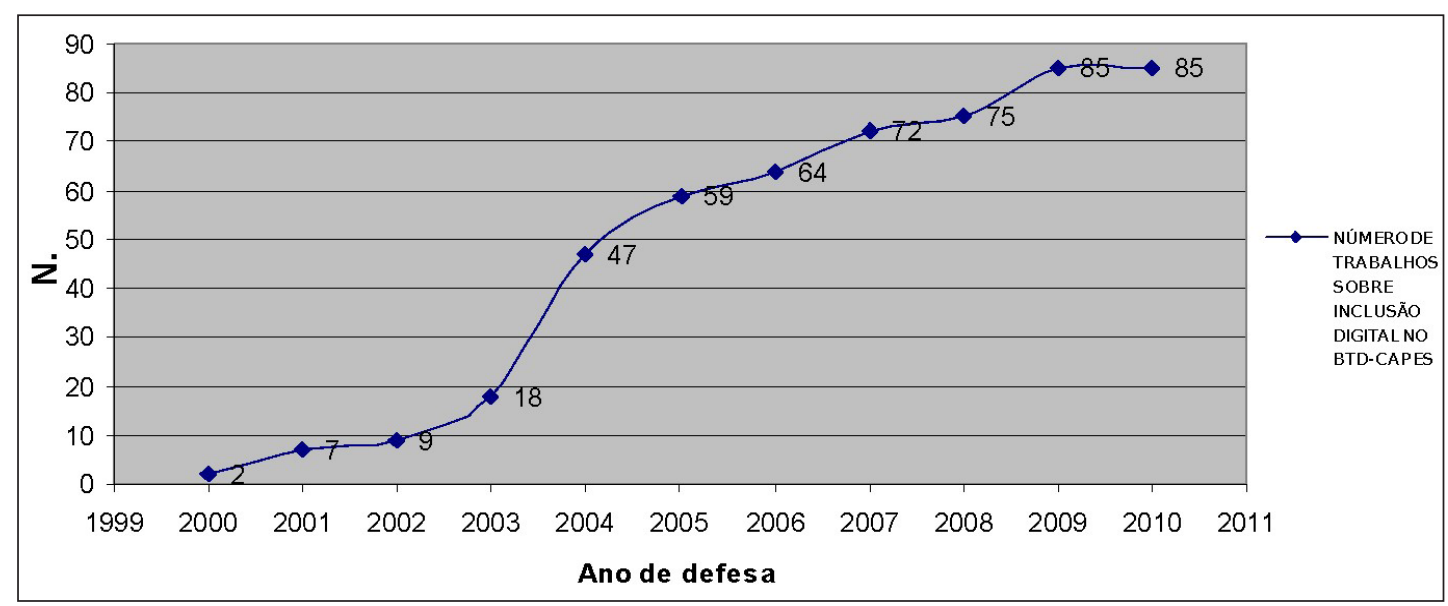

Fonte: Dados da pesquisa.

Figura 1 - Número de trabalhos sobre inclusão digital no Banco de Teses e Dissertações da Capes. 


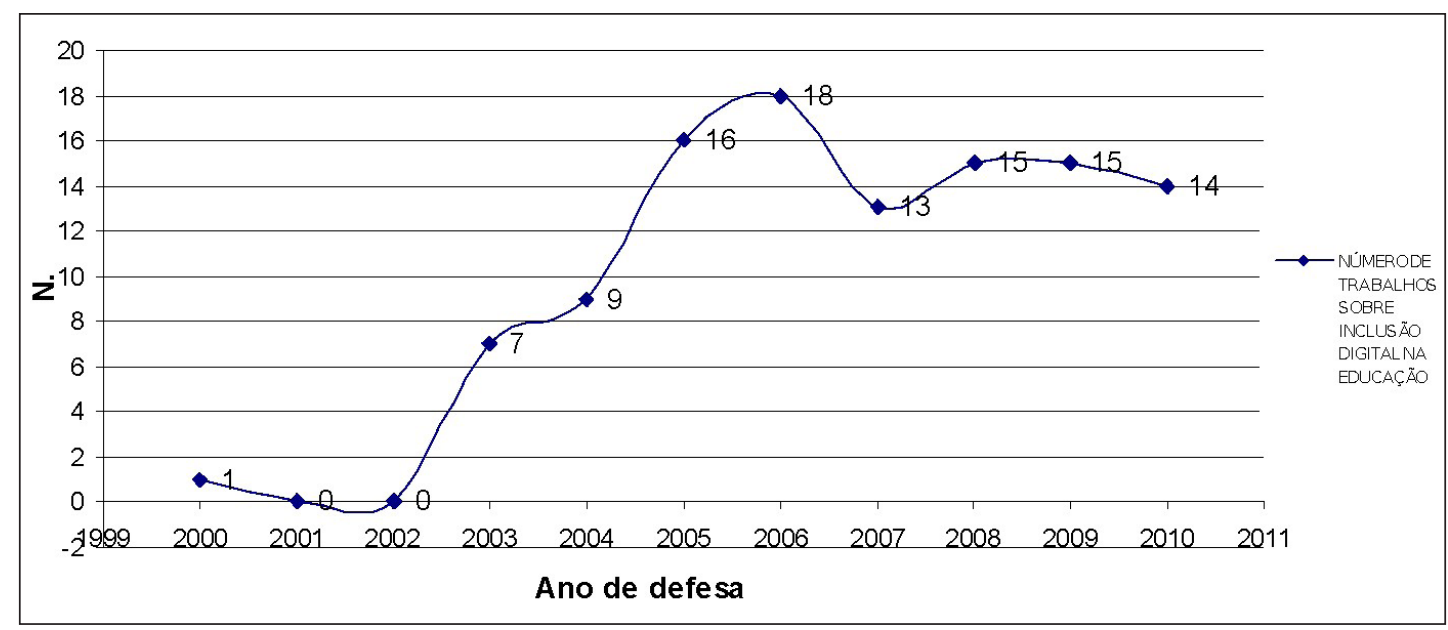

Fonte: Dados da pesquisa.

Figura 2 - Número de trabalhos mapeados sobre inclusão digital na educação no Banco de Teses e Dissertações da Capes.

$\mathrm{Na}$ área de Educação, o tema inclusão digital também apresenta um crescimento constante até o ano de 2006. Assim, torna-se possível perceber o início de uma curva, sendo este um dos aspectos passíveis de problematização, quando se leva em consideração o crescimento da produção do tema nas demais áreas do conhecimento.

Outro ponto de problematização diz respeito à predisposição das discussões nas variadas temáticas que esses estudos sinalizam. Estas são voltadas a inquietações em diferentes contextos e situações-problema da qual a educação (ao longo do estudo está sendo usada a cxa) em sua magnitude é abordada, tendo como pano de fundo, ou permeando o processo, o tema da inclusão digital.

Inicialmente, identificam-se elementos que podem figurar como problemas, para daí se construir um leque variado de questionamentos e apontamentos para a agenda de inclusão digital. E, portanto, permitir que se desenvolva um estudo crítico acerca da produção científica realizada na área de Educação.

\section{UMA APROXIMAÇÃO COM O ESTADO DA ARTE DO CONHECIMENTO SOBRE O TEMA PROPOSTO}

O aprofundamento da revisão de literatura é essencial na busca do enfoque teórico deste estudo. Nesta subseção, apresenta-se o marco teórico e empírico que fundamenta este estudo.

\subsection{O conceito de tecnologia e de informação}

$\mathrm{O}$ conceito de tecnologia entendido neste artigo encontra respaldo nos escritos de Pinto (2005a; 2005b).
Destacam-se as proposições para pensar as tecnologias de Wolton (2012), procurando diminuir a pressão da técnica sobre a comunicação. O teórico da comunicação adverte que o essencial da técnica é de outra ordem, nesse caso, cultural e social, enfocando as relações sociais.

Quem discute inclusão digital na Educação, sobretudo na pós-graduação, apresenta um entendimento sobre as TICs, sinalizando como estas podem ser usadas ou apropriadas pelos sujeitos no processo de inclusão. Desse modo, o conceito de informação por trás das ideias defendidas neste texto é aquele discutido por Capurro e Hjorland (2007), especificamente, no contexto da recuperação da informação. Concorda-se com a junção da semântica com a pragmática na recuperação da informação, proposta pelos autores, em que texto, documento, semântica e significado estão relacionados à informação.

A definição de informação traz influências de Saracevic (2009) na Ciência da Informação, onde esta é compreendida como conjunto estruturado de representações mentais codificadas, socialmente contextualizadas e passíveis de serem registradas em qualquer suporte material e, portanto, comunicadas de forma assíncrona e multidirecionada (SILVA; RIBEIRO, 2008).

\subsection{A compreensão de inclusão e ensino-aprendizagem que respalda o termo inclusão digital na educação}

As discussões sobre inclusão digital na educação estão imersas em um contexto de cibercultura balizada nos trabalhos de Lévy (1994, 1997) e Primo (2007). Próximo a esse debate estão os estudos de Pretto (2002), que tratam do tema formação no contexto de informática na Educação. 
A compreensão de ensino-aprendizagem que envolve este estudo privilegia a perspectiva de mediação e interacionismo com ênfase na abordagem vigotskiana. Assim, destacam-se os trabalhos de Duarte (1993, 2008), Luria, Leontiev e Vigotski (2005). A partir dessa ótica é que se propõe a análise crítica dos trabalhos selecionados no estudo infométrico que se realiza através do desdobramento da pesquisa.

Para se analisar se existe uma tendência do discurso pedagógico contemporâneo (instrumental ou determinista), nos estudos que tratam do tema inclusão digital na Educação, procurou-se um distanciamento do debate habitual sobre tecnologias na educação. Esse questionamento, por sua vez, pode ajudar a explicar se há uma perspectiva de inclusão digital instrumental, que vislumbra a integração da TIC na educação com foco no uso, ou determinista, que entende o fenômeno como o surgimento de um novo modo de ação na Educação. Para observar essa questão, os estudos de Gómez Galán (2012), que tratam sobre o discurso pedagógico acerca da tecnologias na Educação e sobre as correntes de investigação em tecnologia educativa, são trabalhos relevantes para a discussão aqui proposta.

Os estudos de Ribeiro (1999), Demo (2002), Freitas (2002), Silva Filho (2003), Silveira e Cassino (2003), Neves e Couto (2012), Neves (2010), Dijk (2005 e 2006), Warschauer (2003, 2006a, 2006b, 2007), Assumpção e Mori (2007), Buzato (2007), Bonilla e Oliveira (2011) e Leite (2011) também sustentam de forma interessante o tema proposto. As discussões nessa literatura, até o momento, selecionadas sobre inclusão digital, problematizam pelo viés das políticas públicas. Somandose a essas destacam-se, ainda, os textos organizados por Hetcowiski (2008). A noção que permeia, ou embasa, o tema pode influenciar na defesa do debate que direciona o assunto na Educação.

A compreensão de inclusão que respalda a concepção de inclusão digital debatida neste artigo está balizada no conceito de integração social (CASTEL, 1998), na qual os indivíduos estão incluídos e excluídos simultaneamente no mundo, no sistema, dependendo da perspectiva do objeto de desejo. Essa concepção de inclusão entende como base os vínculos relacionais que os sujeitos podem desempenhar em seus diferentes papéis.

Outro aspecto que é considerado, nessa perspectiva de inclusão, é que todos estão integrados no sistema, sendo que uns estão mais próximos do centro capitalista e outros mais nas bordas, a exemplo de moradores de rua, detentos e deficientes não assistidos. Contudo, mesmo esses indivíduos à margem pressionam e influenciam todo o sistema. O que também encontra sustentação na discussão realizada por Demo (2002), quando este observa o charme conferido ao conceito exclusão social. Esse debate, que envolve o par dialético "exclusão e inclusão", assim como a perspectiva de integração social, tem sido de extrema importância à área das ciências sociais.

Percebendo isso, entende-se a relevância de discutir esses conceitos à luz de Sorj (2003, 2006). Quando necessário, visitam-se as teorias de sociedade em rede, de Castells (2000). A perspectiva sociológica não constitui o eixo principal do estudo, mas, certamente, lubrifica as engrenagens da discussão desta análise. Afinal, parte-se da premissa de que todos os trabalhos que discutem a inclusão possuem como prerrogativa o acesso, a inserção ou a emancipação dos sujeitos em um novo paradigma implementado por conta de inovações e tecnologias nas suas inter-relações sociais. Essas transformações alteram a cultura e as relações sociais, e, em um movimento dialético, estruturam e são estruturadas pela sociedade.

A grade de leitura que culminou na problematização da pesquisa em andamento é mais ampla que a apresentada neste artigo. Destacam-se aqui as referências que são trabalhadas mais diretamente na discussão dos pontos abordados por este estudo, especialmente aquelas que fornecem subsídios metodológicos para qualificá-lo.

\section{Políticas públicas de INCLUSÃO DIGITAL NA EDUCAÇÃO: A AGENDA}

Acredita-se que a agenda de inclusão digital na Educação está ligada às políticas públicas. Elementos dessas políticas são abstraídos para discutir questões relacionadas à infraestrutura de acesso, treinamento e formação. No Brasil, as discussões acerca da inclusão digital começam a partir da difusão do Livro Verde da Sociedade da Informação. O lançamento do Programa Sociedade da Informação no Brasil (Socinfo) teve início no ano de 1999, em Brasília-DF. A agenda de lançamentos e discussões das linhas de ação do programa se estendeu até o ano 2000, passando por várias capitais.

Vale destacar que os assuntos que dizem respeito à inclusão digital tiveram início com o Socinfo. A partir desse marco, os ministérios do governo brasileiro, ligados a essa área, passaram a ter preocupações com relação à infraestrutura de acesso e à inserção das organizações públicas e privadas na nova ordem de tecnologias de informação e comunicação mundial que se instalava. O desenvolvimento de variados programas e projetos, citados aqui como exemplos, para acesso das populações às TICs, vem sendo, desde então, oriundo de linhas de ação dos ministérios do Planejamento, das Telecomunicações, da Ciência Tecnologia e Inovação e da Educação (MEC).

O MEC já havia lançado, em 1997, o Programa Nacional Informática na Educação (Proinfo), que continha em seu escopo ações como a Rádio Escola, a 
TV Escola e o DVD Escola. Esse programa passou por uma reformulação em 2007, incorporando novas ações e formulação de objetivos, a exemplo da adequação e uso do software livre e de projetos com previsão de acesso para o urbano e para o rural.

De acordo com Free Software Foudation (2013), entende-se por software livre aquele que respeita a liberdade e o senso de comunidade dos usuários. Neste, os usuários possuem a liberdade de executar, copiar, distribuir, estudar, mudar e melhorar o software. Assim sendo, software livre é uma questão de liberdade, não de preço.

O Programa Um Computador por Aluno (ProUCA), implementado em 2010, é mais um programa ligado às ações de informática na educação promovido pelo MEC. O governo federal forneceu 150 mil laptops do programa a 300 escolas estaduais no projeto piloto (QUARTIERO; BONILLA; FANTIN, 2015). Outras políticas do MEC que perpassam a inclusão digital, e que, consequentemente, interessam a este estudo, são o Projeto de Educação Digital (Tablet Educacional); o Programa Banda Larga nas Escolas, criado em 2009, a partir de um acordo com a OI Telecomunicações; e o Plano Nacional de Banda Larga (PNBL), com a reativação da Telebrás em 2010.

Para falar de política pública no campo da Educação, não se pode deixar de citar o Manifesto dos Pioneiros da Educação Nova, que serviu de inspiração para as principais leis na Educação brasileira. Criado em 1932, trata-se de um dos principais documentos que conferiram direcionamento político por meio da definição do que seriam as principais metas educacionais.

Outro marco diz respeito às Leis de Diretrizes e Bases da Educação Nacional (LDB), a saber: Lei no 4.024, de 20 de dezembro de 1961, que fixa as Diretrizes e Bases da Educação Nacional; Lei no 5.692, de 11 de agosto de 1971, que fixa as Diretrizes e Bases para o Ensino de 1음 e $2^{\circ}$ graus, e confere outras providências; e Lei no 9.394 , de 20 de dezembro de 1996, que estabelece as Diretrizes e Bases da Educação Nacional.

Para a educação sair de um período de autoritarismo e centralização - herdado do seu passado histórico - e passar para um nível concreto de maior participação de outros segmentos da estrutura do Estado, assim como da sociedade, no estabelecimento e desenvolvimento dessas políticas, foi necessário um complexo exercício na configuração dos sistemas educacionais.

Como é possível perceber, as políticas aqui citadas possuem ligação implícita com períodos e linhas de estudo, influenciando o aparecimento de pesquisas com temas nesse campo.

O tema inclusão digital é tratado na Educação sob várias abordagens próprias de questionamentos que emergem entre sujeitos e recursos na ação educativa. Este estudo identificou que o enfoque dos pesquisadores dessa área parte de preocupações relacionadas a:

- formação de professores;

- processos educacionais;

- políticas públicas;

- currículo;

- acessibilidade.

Esses indicadores (clusters), levantados no estudo macro, são compreendidos como categorias em que são agrupadas as principais perspectivas dos pesquisadores da área de Educação.

A identificação desses clusters permite elaborar questionamentos, sendo um dos pontos que se procurou traduzir em questões de pesquisa, visando a clarificar a agenda da inclusão digital na Educação.

\section{À GUISA DE CONCLUSÃo}

A pesquisa que se empreendeu vislumbra a oportunidade de que se possa estudar com maior profundidade o tema da inclusão digital, desta vez, na Educação. Para tanto, além do levantamento da produção acadêmica até agora defendida e registrada no Banco de Teses e Dissertações da Capes, procurou-se associar métodos específicos de pesquisa e análise de dados pouco explorados na área de Educação.

Compreender como e por que os pesquisadores dessa área constroem suas perguntas de pesquisa e abordam o tema inclusão digital representa um problema relevante para se traçar uma agenda de pesquisa sobre tal temática.

Os resultados parciais apresentados neste artigo fazem parte de uma pesquisa mais ampla e profunda, pois mapeia de maneira exaustiva o Banco de Teses e Dissertações da Capes com o intuito de analisar como a inclusão digital vem sendo estudada e fundamentada, ao longo dos anos 2000, no que concerne a sua agenda de pesquisa para a Educação brasileira.

Ressalta-se que o quantitativo de trabalhos defendidos sobre o tema nas diversas áreas do conhecimento continua em crescimento. Em comparação, no mesmo período, na área de Educação, é possível perceber uma curva no cálculo da idade média da produção (defesas sobre o tema).

Este artigo também revela que os principais clusters identificados são formação de professores, processos educacionais, políticas públicas, currículo e acessibilidade. Esses campos são amplamente discutidos na Educação, constituindo-se nas principais linhas de trabalho/estudo para os pesquisadores que tratam do tema nessa área.

\section{REFERÊNCIAS}

ASSUMPÇÃO, Rodrigo; MORI, Cristina. Inclusão digital: 
discursos, práticas e um longo caminho a percorrer. In: KNIGHT, Peter Titcomb; FERNANDES, Ciro Campos Christo; CUNHA, Maria Alexandra (Org.). E-Desenvolvimento no Brasil e no mundo: subsídios e programas e-Brasil. São Paulo: Yendis, 2007.

BONILLA, Maria Helena Silveira; OLIVEIRA, Paulo Cezar Souza de. Inclusão digital: ambiguidades em curso. In: BONILLA, Maria Helena Silveira; PRETTO, Nelson de Luca. Inclusão digital: polêmica contemporânea. Salvador: Edufba, 2011.

BRASIL. Sociedade da informação: ciência e tecnologia para a construção da sociedade da informação no Brasil. Brasília: Ministério da Ciência e Tecnologia, 2000.

BUZATO, Marcelo El Khouri. Entre a fronteira e a periferia: linguagem e letramento na inclusão digital. 2007. 285 f. Tese (Doutorado em Linguística Aplicada) - Instituto de Estudos da Linguagem, Universidade Estadual de Campinas, Campinas, 2007.

CAPURRO, Rafael; HJORLAND, Birger. O conceito de informação. Perspectiva em Ciência da Informação, v. 12, n. 1, p. 148-207, 2007. http://dx.doi.org/10.1590/S141399362007000100012

CASTEL, Robert. As metamorfoses da questão social: uma crônica do salário. Tradução de Iraci Poleti. Petrópolis: Vozes, 1998.

CASTELLS, Manuel. A sociedade em rede. Tradução de Roneide Venancio Majer. São Paulo: Paz e Terra, 2000.

DEMO, Pedro. Charme da exclusão social. Campinas: Autores Associados, 2002.

DIJK, Jan Van. The network society: social aspects of new media. 2. ed. London: Sage Publication, 2006.

DIJK Jan Van. The deepening divide: inequality in the information society. Thousand Oaks: Sage Publicaions, 2005.

DUARTE, Newton. Sociedade do conhecimento ou sociedade das ilusões? Campinas: Autores Associados, 2008.

DUARTE, Newton. A individualidade para-si: contribuição a uma teoria histórico-social da formação do indivíduo. Campinas: Autores Associados, 1993.

FREITAS, Luiz Carlos de. A internalização da exclusão. Educ. Soc., Campinas, v. 23, n. 80, p. 299-325, set. 2002.

GÓMEZ GALÁN, José. Corrientes de investigación en tecnología educativa. In: GÓMEZ GALÁN, José; SANTOS, Gilberto Lacerda (Org.). Informática y telemática en la educación. Brasília: Liber Livro, 2012. p. 105-157.

GUEDES, Vania; BORSCHIVER, Suzana. Bibliometria: uma ferramenta estatística para a gestão da informação e do conhecimento, em sistemas de informação, de comunicação e de avaliação científica e tecnológica. In: ENCONTRO NACIONAL DE CIÊNCIA DA INFORMAÇÃO, 6., 2005, Salvador. Anais eletrônicos... Salvador: ICI/UFBA, 2005.

HETKOWSKI, Tania Maria (Org.). Políticas públicas e inclusão digital. Salvador: Edufba, 2008.

LEITE, Ligia Silvia. Mídia e a perspectiva da tecnologia educacional no processo pedagógico contemporâneo. In: FREIRE, Wendel et al. Tecnologia e educação: as mídias na prática docente. Rio de Janeiro: Wak, 2011.
LÉVY, Pierre. Cyberculture. Paris: Éditions Odile Jacob, 1997. LÉVY, Pierre. L'intelligence colletive. Pour une anthopologie du cyberspace. Paris: Éditions La Découvert, 1994.

LURIA, Alexandre Romanovich; LEONTIEV, Alexis; VYGOTSKY, Lev. Psicologia e pedagogia: bases psicológicas da aprendizagem e do desenvolvimento. São Paulo: Centauro, 2005.

NEVES, Bárbara Coelho; COUTO, Edvaldo Souza. Convergência de recursos e mediação para inclusão digital: casos baianos. Educar em Revista, n. 46, p. 245-257, out./dez. 2012. ISSN 0104-4060. http://dx.doi.org/10.1590/S010440602012000400017

NEVES, Bárbara Coelho. Análise das políticas de informação: sociedade da informação com foco na inclusão digital do global ao local. Revista Iberoamericana de Ciencia, Tecnología y Sociedad, v. 5, n. 15, p. 111-131, 2010.

PINTO, Álvaro Vieira. O conceito de tecnologia. Rio de Janeiro: Contraponto, 2005a. 1 v.

PINTO, Álvaro Vieira. O conceito de tecnologia. Rio de Janeiro: Contraponto, 2005b. 2 v.

PRETTO, Nelson de Luca. Formação de professores exige rede! Revista Brasileira de Educação, Rio de Janeiro, n. 20, p. 121-131, maio/ago. 2002.

PRIMO, Alex. Interação mediada por computador: comunicação, cibercultura, cognição. Porto Alegre: Sulina, 2007.

QUARTIERO, Elisa Maria; BONILLA, Maria Helena Silveira; FANTIN, Monica (Org.). Projeto UCA: entusiasmos e desencantos de uma política pública. Salvador: Edufba, 2015.

RIBEIRO, Marlene. Exclusão: problematização do conceito. Educação e Pesquisa, São Paulo, v. 25, n. 1, p. 23-49, jan./ jun. 1999.

SARACEVIC, Tefko. Information science. In: BATES, Marcia; MAACK, Mary Niles (Ed.). Encyclopedia of Library and Information Science. New York: Taylor \& Francis, 2009.

SILVA, Armando Malheiro; RIBEIRO, Fernanda. Das ciências documentais à ciência da informação: ensaio epistemológico para um novo modelo curricular. Porto: Afrontamento, 2008.

SILVA FILHO, Antônio Mendes da. Os três pilares da inclusão digital. Revista Espaço Acadêmico, n. 24, maio 2003.

SILVEIRA, Sérgio Amadeu. Software livre: a luta pela liberdade do conhecimento. São Paulo: Fundação Perseu Abramo, 2004.

SILVEIRA, Sergio Amadeu; CASSINO, João (Org.) Software livre e inclusão digital. São Paulo: Conrad, 2003.

FREE SOFTWARE FOUNDATION. O que é software livre. A definição de software livre, GNU, 2013. Disponível em: <gnu@ gnu.org>. Acesso em: 23 nov. 2015.

SORJ, Bernardo. A nova sociedade brasileira. 3 ed. Rio de Janeiro: Jorge Zahar, 2006.

SORJ, Bernardo. Brasil@povo.com: a luta contra a desigualdade na sociedade da informação. Brasília, DF: UNESCO, 2003.

VANTI, Nádia Aurora Peres. Da bibliometria à webometria: uma exploração conceitual dos mecanismos utilizados para medir o registro da informação e a difusão do conhecimento. Ciência da Informação, Brasília, v. 31, n. 2, p. 152-162, maio/ ago. 2002. 
VANTI, Nádia Aurora Peres. Os links e os estudos webométricos. Ciência da Informação, Brasília, v. 34, n. 1, p. 78-88, jan./abr. 2005.

WARSCHAUER, Mark. Technology and social inclusion: rethinking the digital divide. Massachusetts: MIT press, 2003.

WARSCHAUER, Mark. Tecnologia e inclusão social: a exclusão digital em debate. São Paulo: Senac, 2006a.

WARSCHAUER, Mark. Learning the digital age. Educational Leaderschip, London, v. 63, n. 4, p. 34-38, dec. 2005/-jan. 2006b.
WARSCHAUER, Mark. Laptops and literacy: learning in the wireless classroom. New York: Teaches College Press, 2007.

WOLTON, Domique. Internet e depois? Uma teoria critica das novas mídias. Porto Alegre: Sulina, 2008.

\section{Nota}

1 A infometria é um método de pesquisa utilizado para a captura dos dados sobre esta temática. Utilizou-se para a mineração o VantagePoint.

Artigo recebido em setembro 2015.

Aprovado em dezembro 2015. 